\title{
Modeling and Simulation of Sierpinski Pentagon Fractal Antennas
}

\author{
Malek A.H. Muhi* and Mohammed A.Z. Habeeb \\ Department of Physics, College of Science, Al-Nahrain University, Baghdad-Iraq. \\ E-mail: malik_muhi@yahoo.com.
}

\begin{abstract}
Measurements of fractal shaped antennas are not enough to understand their physical behavior and there are antenna characteristics that can not be obtained by present day measurement techniques. Therefore, in the present work, the Ansoft HFSS finite element electromagnetic computer package was used to model, simulate and analyze the Sierpinski pentagon monopole microstrip patch fractal antenna and its complementary. The simulation results for both antennas show good matching with their feeds in the frequency range $(2 \mathrm{GHz}-12 \mathrm{GHz})$ and indicate that they can operate in the UMTS (2000 MHz-2200 MHz), Bluetooth $(2400 \mathrm{MHz}-2480 \mathrm{MHz})$, WLAN $(2.4 \mathrm{GHz})$ and HIPERLAN $(5.2 \mathrm{GHz})$ bands. Also, the computed results show, in general, good agreement with measured data for the S-parameter and radiation patterns.
\end{abstract}

Keywords: fractal geometry, fractal antennas, Sierpinski pentagon, computer simulation.

\section{Introduction}

It is well-known that problems accompanying antennas are related to the requirement of operation at a single frequency, limited directivity of their radiation patterns, their physical size and their efficiency or gain. In addition, specific problems have been observed in microstrip patch type antennas, such as narrow bandwidth and difficulty in achieving the required polarization. To overcome some of these problems, a newly discovered branch of modern mathematics called "fractal geometry" [1] has been utilized in the design of antennas. Antennas constructed this way were coined "fractal antennas" by Nathan Cohen in 1995 [2]. Space-filling, self-similarity and fractional dimension are common properties of fractal shapes that make fractal shaped antennas lend themselves easily for miniaturization, multiband performance, improved pattern directivity and better efficiency [3]. It is also well-known that measurements of fractal shaped antennas are not enough to understand completely their physical behavior and there are fractal shaped antenna characteristics that could not be obtained by present day measurement techniques. Therefore, computer simulations are considered useful to supplement this aspect of the problem.

Indeed, hundreds of research works have been published on fractal shaped antennas [4, $5,6]$. Fractal antennas studied include specific shapes, such as the Koch curve, Sierpinski triangle, Hilbert curve, Minkowski curve and Peano curve [4]. More recently, two novel fractal shaped monopole microstrip patch antennas were suggested, construlted and measured by $M$. Naghshvarian-Jahromi and N. Komjani [7]. Their geometry is based on the Sierpinski pentagon fractal and its complementary fractal.

The aim of the present work is to supplement the laboratory measurements for these novel fractal shaped antennas by computer simulation to confirm and to further understand their behaviour and novel properties. To this end, the computer software Ansoft HFSS (High Frequency Structure Simulator) [8] will be used to perform this simulation work and to the best of our knowledge this is the first time this type of simulation work is performed for these two fractal shaped antennas.

\section{The HFSS Simulation Technique}

The computer software Ansoft HFSS [8] is based on the finite element method (FEM) which is applied to differential equations with boundary values to obtain an approximate solution. In this software, the geometrical model is divided into large number of tetrahedral elements. The collection of tetrahedral elements represents the mesh of the FEM. At points inside each tetrahedron, the value of a vector field quantity is interpolated 
from its values at the vertices of the tetrahedron. The HFSS software has an advantage for generating the mesh. It employs the method of adaptive analysis, in which the mesh is automatically refined in complicated or critical regions [8]. After the HFSS software divides the geometrical region into elements, there are three steps to calculate the electromagnetic structure with ports as follows:

(i) Computation of the modes at each port of the structure that is supported by a transmission line having the same crosssection as the port.

(ii) Computation of the full electromagnetic field pattern inside the structure.

(iii) Computation of the generalized S-matrix from the amount of reflection and transmission that occurs [8].

The excitation field $\vec{E}(x, y, z, t)$ is the field associated with traveling waves propagating along the guiding device to which the port is connected, or [8]

$$
\vec{E}(x, y, z, t)=\operatorname{Re}\left[\vec{E}(x, y) e^{j \omega t-\gamma z}\right]
$$

where $\vec{E}(\mathrm{x}, \mathrm{y})$ is a phasor field quantity, $\gamma$ is the complex propagation constant, and $\omega$ is angular frequency. Then, the HFSS software solves Maxwell's equations in terms of the S-matrix which represents the fraction of power associated with field excitation being transmitted or reflected at each port. From the S-matrix many parameters can be calculated.

When radiation fields are calculated by the HFSS software, this software needs a radiation surface (boundary condition) to be defined surrounding the device. This surface has the values of the fields. Typically, this boundary has two regions corresponding to the near- and far- fields [8]. In general, the boundary region can be represented by a closed surface on which the electric field may be written as [8]:

$$
\begin{aligned}
& \vec{E}(x, y, z)=\int_{s}\left(<j \omega \mu_{o} \vec{H}_{\text {tan }}>\mathcal{G}+<\vec{E}_{\text {tan }} \times\right. \\
& \left.\vec{\nabla} \mathcal{G}>+<\vec{E}_{\text {normal }} \cdot \vec{\nabla} \mathcal{G}>\right) d s
\end{aligned}
$$

where $\vec{H}_{\text {tan }}$ is the component of the magnetic field that is tangential to the surface, $\vec{E}_{\text {normal }}$ is the component of the electric field that is normal to the surface, $\vec{E}_{\text {tan }}$ is the component of the electric field that is tangential to the surface, $\mu_{o}$ is the relative permeability of the free space and $\mathcal{G}$ is the free space Green's function given by [8]:

$\mathcal{G}=\frac{e^{-j v_{0}\left|\vec{r}-\vec{r}^{\prime}\right| \sqrt{\mu_{r} \varepsilon_{r}}}}{\left|\vec{r}-\vec{r}^{\prime}\right|}$

where $v_{o}$ is the free space wave number, $\mu_{r}$ is the relative permeability of the medium and $\vec{r}, \vec{r}^{\prime}$ represent position vectors of field and source points on the radiation surface, respectively [8].

Even though the HFSS software was not originally designed to solve electromagnetic problems for fractal geometries, it has been used for such geometrics, especially fractal shaped antenna problems, with success [9-12]. Problems that need to be overcome in such fractal shaped antenna HFSS simulations include the generation of the fractal antenna geometry suitable for feeding into the HFSS software, the choice of the suitable numerical mesh that guarantees stability and convergence of the FEM solutions and the overcoming of computing time and storage demands associated with fine meshes needed for fractal geometries. Some of these problems are discussed in the next sections.

\section{Generation of the Sierpinski Pentagon Fractal}

The Sierpinski pentagon fractal is generated by an iterated function system (IFS) process [13]. It begins with a pentagon $\mathbb{P}_{0}$ representing an initiator. In the second stage, the five small copies of the pentagon are obtained when scaling down by a factor. Then, each one of the small pieces is translated from its vertex to five vertices of the large pentagon to fit these small pieces inside large one. After that, the large piece is subtracted from the small pieces. Fig.(1) illustrates the structure of $\mathbb{P}_{1}$ that represents the generator. To construct the next iteration, this processes is repeated on the new set $\mathbb{P}_{1}$. In the same figure, some iterations of Sierpinski pentagon are also shown. 


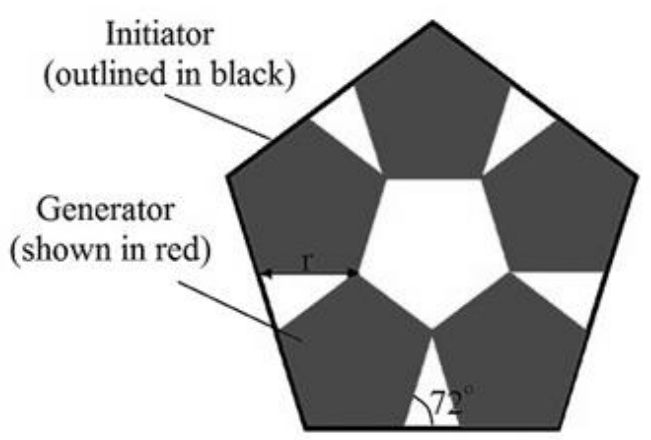

(a) The construction of the initiator

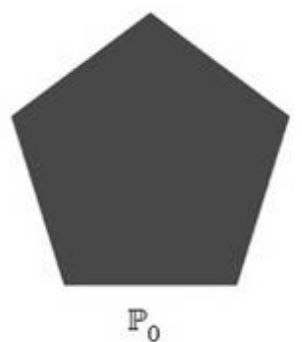

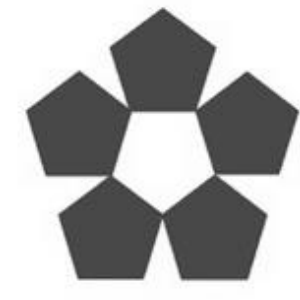

$\mathbb{P}_{1}$

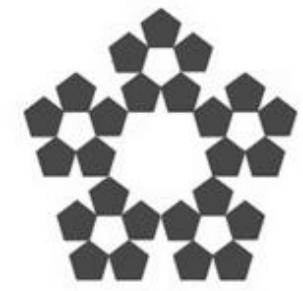

$\mathbb{P}_{2}$

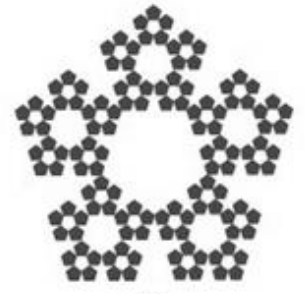

$\mathbb{P}_{3}$

(b) The first three iterations

Fig. (1) The Sierpinski Pentagon [13].

The scale factor for transformation function is $r=\frac{3-\sqrt{5}}{2}$ [13]. So that the IFS for Sierpinski pentagon is the following [13]

$$
\left.\begin{array}{rl}
f_{1}(x) & =\left[\begin{array}{cc}
0.382 & 0 \\
0 & 0.382
\end{array}\right] x \\
f_{2}(x) & =\left[\begin{array}{cc}
0.382 & 0 \\
0 & 0.382
\end{array}\right] x+\left[\begin{array}{c}
0.618 \\
0
\end{array}\right] \\
f_{3}(x) & =\left[\begin{array}{cc}
0.382 & 0 \\
0 & 0.382
\end{array}\right] x\left[\begin{array}{c}
0.809 \\
0.588
\end{array}\right] \\
f_{4}(x) & =\left[\begin{array}{cc}
0.382 & 0 \\
0 & 0.382
\end{array}\right] x+\left[\begin{array}{c}
0.309 \\
0.951
\end{array}\right] \\
f_{5}(x) & =\left[\begin{array}{cc}
0.382 & 0 \\
0 & 0.382
\end{array}\right] x+\left[\begin{array}{c}
-0.191 \\
0.588
\end{array}\right]
\end{array}\right\}
$$

As seen, there are five maps for this hyperbolic IFS, with each ratio being greater than one. Therefore, the fractal dimension is $[1,13] D_{f}=\frac{\log (5)}{\log \left(\frac{2}{3-\sqrt{5}}\right)}=1.67228$.

The complementary Sierpinski pentagon fractal is obtained by applying the complementary of the IFS of Eqs. (4).

The results of applying the IFS map described above and its complementary are fed into the HFSS software as the first step for generating the two antenna geometries studied in the present work.

\section{Computations, Results and Discussion}

Both antenna models, employed as monopole microstrip patches that are shown in Fig.(2), have the same parameters except for the form of their patches. The computations for the S-parameter in the HFSS software for the Sierpinski pentagon model were performed in the frequency range $(0.5-13) \mathrm{GHz}$, and for the complementary Sierpinski pentagon in the frequency range $(0.5-15) \mathrm{GHz}$. The measured data for the two antenna models have been obtained in the frequency range $(0.5-20) \mathrm{GHz}$ [7]. The computed and measured S-parameter results for the two fractal shaped antennas are shown in Fig.(3) for comparison purposes. As seen in Fig.(3), the HFSS software is unable to analyze frequencies higher than $13 \mathrm{GHz}$ for Sierpinski pentagon antenna and higher than $15 \mathrm{GHz}$ for complementary Sierpinski pentagon antenna. This is due to the fact that the HFSS software creates very small meshes on both antennas to analyze them for the highest frequencies (e.g., more than $13 \mathrm{GHz}$ ). Furthermore, the adaptive meshes will be very complex because the fractals in these antennas have complicated shapes. 

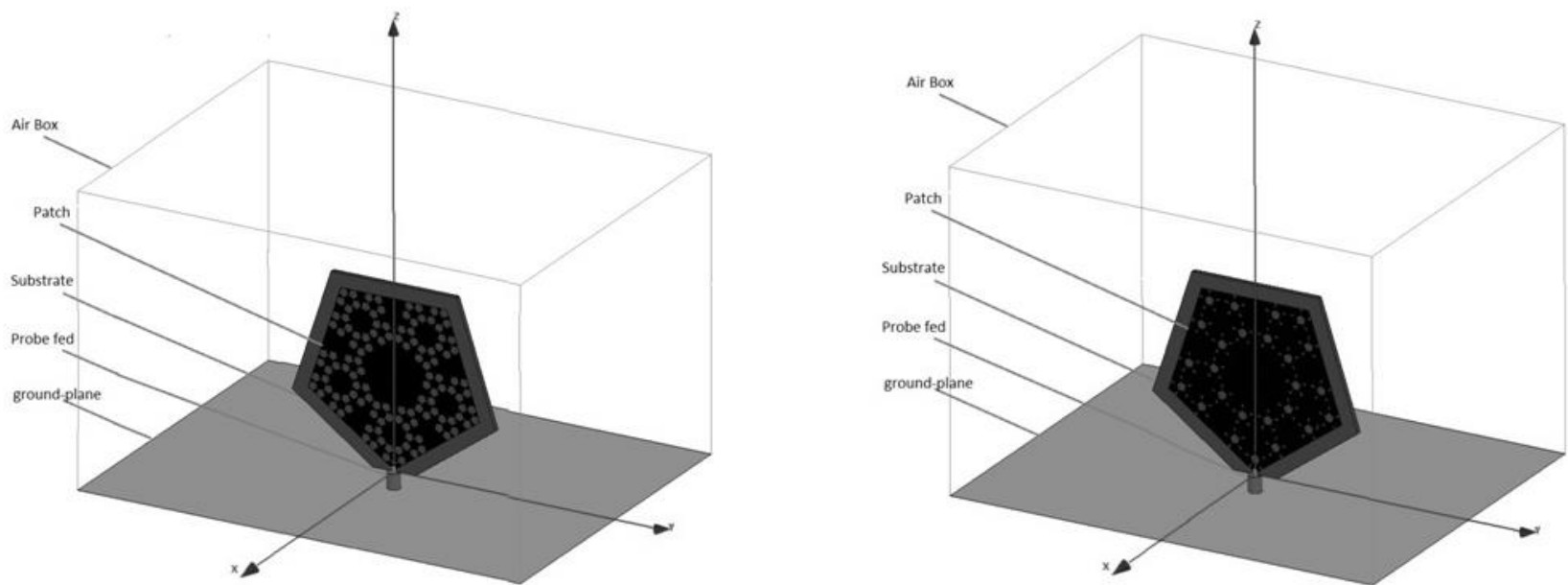

Fig. (2) 3D layouts for monopole fractal shaped antenna as generated by HFSS; (left) Sierpinski pentagon patch and (right) complement Sierpinski pentagon patch.

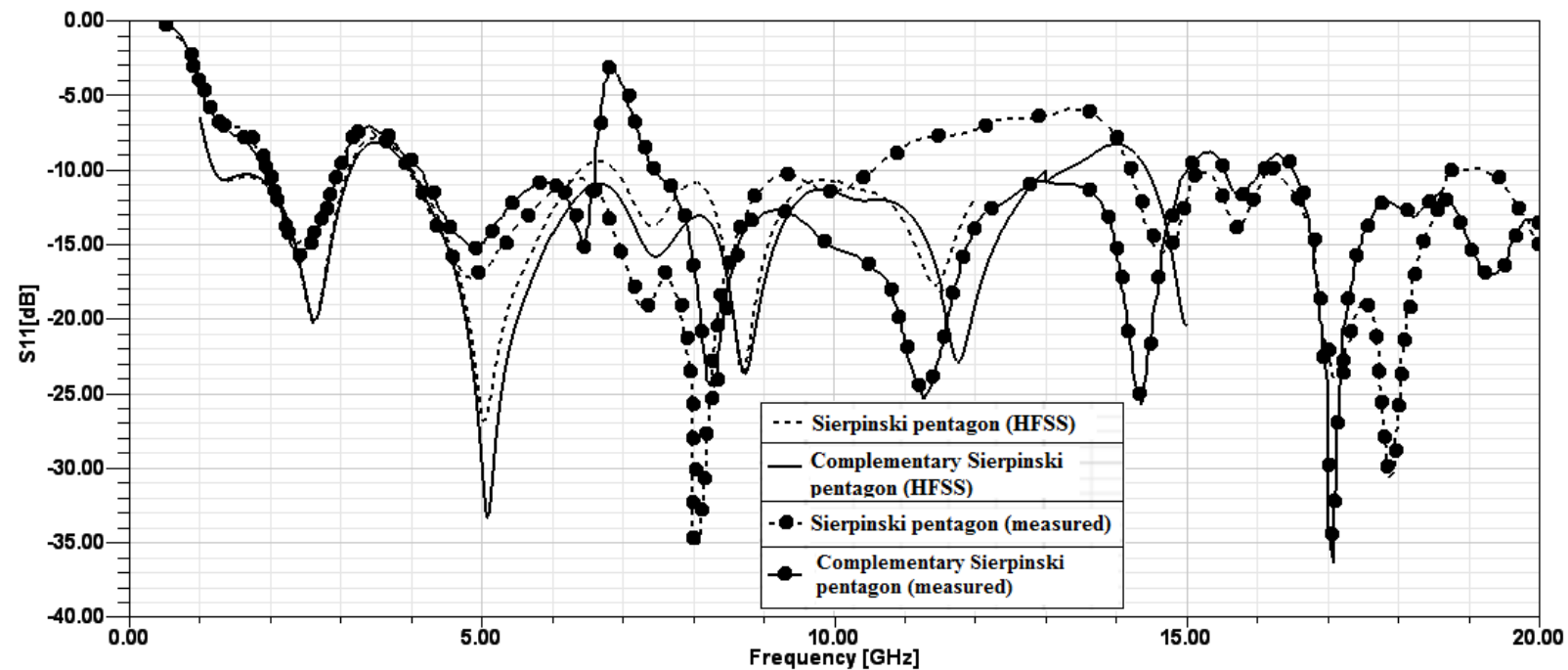

Fig. (3) Behavior of the measured [7] and computed S-parameter $\left(S_{11}\right)$ as a function of frequency for both the Sierpinski pentagon and the complementary Sierpinski pentagon microstrip patch fractal shaped antennas in the HFSS software.

Fig.(4) shows the variation of the computed voltage standing wave ratio (VSWR) with frequency for these two fractal shaped antennas to illustrate their degree of matching with feeds. As seen from this figure, nearly all frequencies are in good matching having $\mathrm{VSWR}<2$. Fig.(5) presents the variation of the computed scattering coefficient $\left(\mathrm{S}_{11}\right)$ as a Smith chart. One notices that the curves have many loops which distribute around the centre of the Smith chart. Each one of these loops represents a specific band in a rectangular plot of the S-parameter as shown in Fig.(3). This result indicates the good matching between antennas and their feeds at frequencies in the range $(0.5$ 15) GHz. No measured data are available for both the VSWR and the Smith chart for these two fractal shaped antennas are available to compare with at present and these computed results represent and addition to the understanding of the behaviour of these two antennas. 


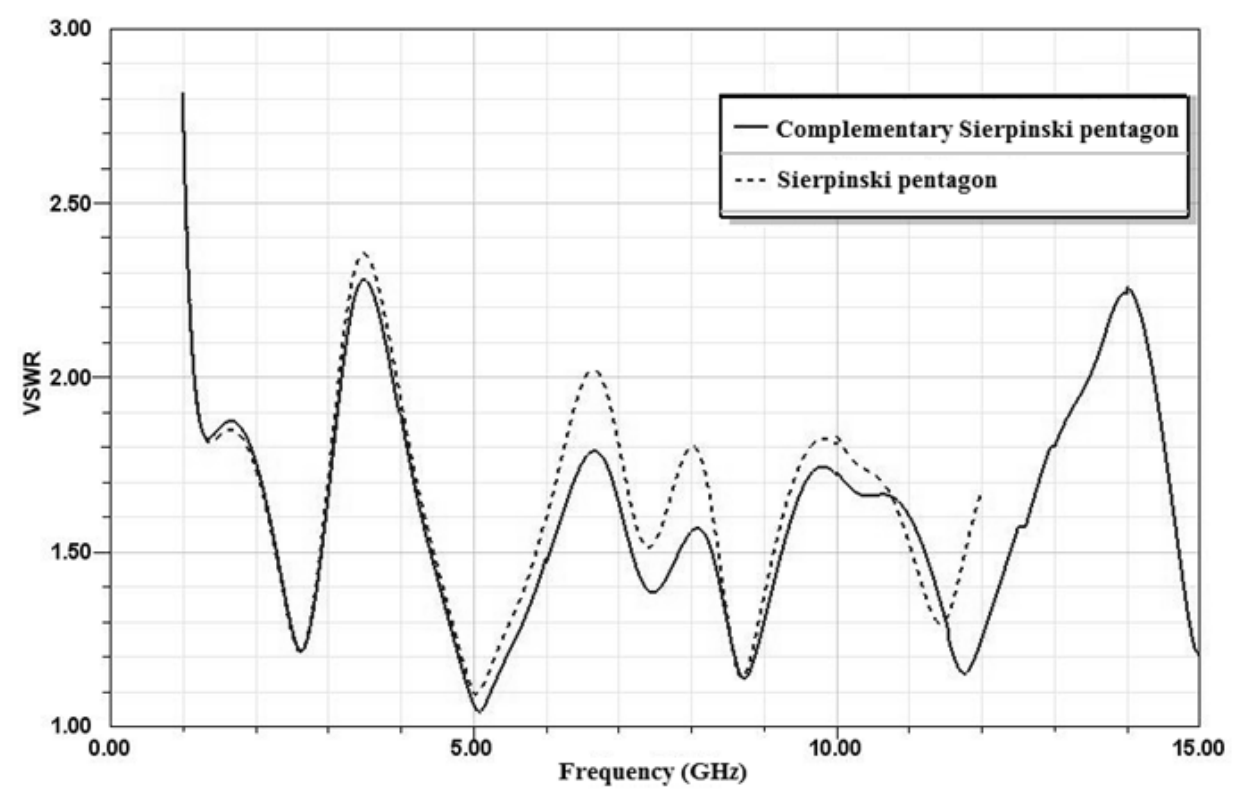

Fig. (4) Behavior of the computed VSWR as a function of frequency for both the Sierpinski pentagon and the complementary Sierpinski pentagon fractal shaped antennas in the HFSS software.

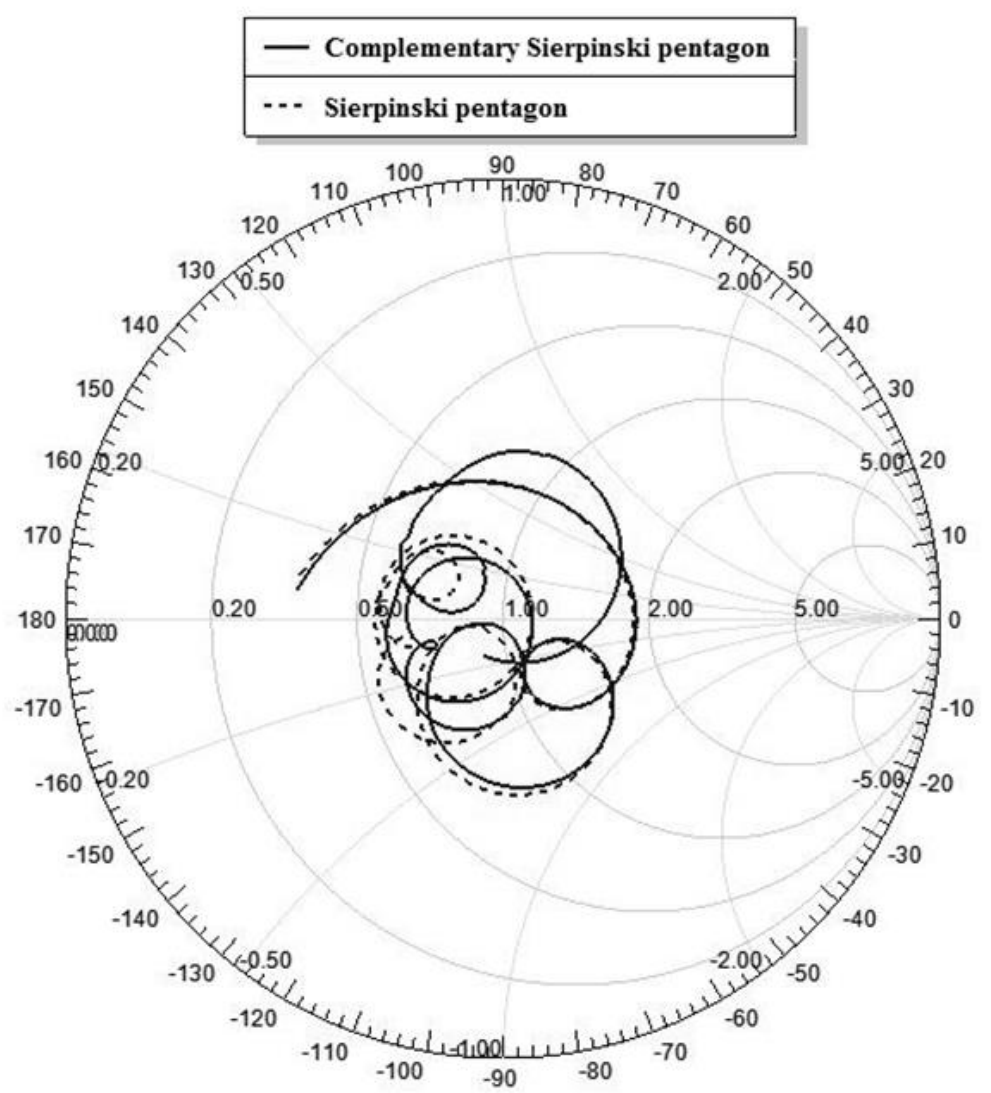

Fig. (5) The two curves of the computed Smith chart for both the Sierpinski pentagon and the complementary Sierpinski pentagon fractal shaped antennas obtained from the HFSS software.

For comparison with measured data in ref. [7], the radiation patterns were computed using the HFSS software at $2.4 \mathrm{GHz}$ and 5.2 GHz representing WLAN and HIPERLAN bands respectively. E-plane and H-plane radiation patterns for the two fractal shaped antennas at these frequencies are presented in Figs. (6) and (7). As seen from these figures, the shapes of the computed and experimental 
curves have the same behavior. Otherwise, the magnitudes.

Otherwise, the magnitudes of the computed curves for the Sierpinski pentagon are smaller than the magnitude of the complementary Sierpinski pentagon curves when they are represented in deci Bell $(\mathrm{dB})$ units at each frequency. These differences are attributed to the differences of the values of the S-parameters.

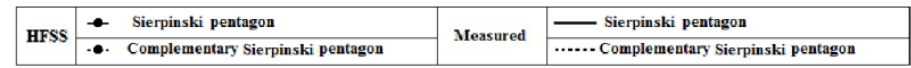
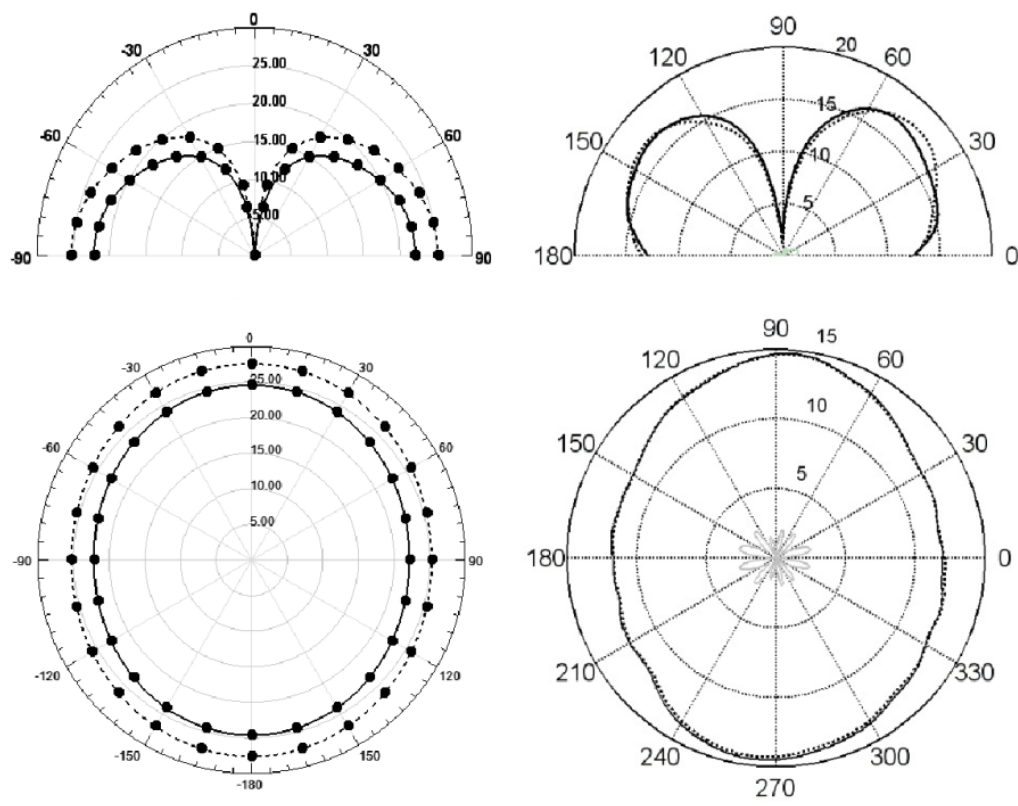

Fig. (6) Measured [7] and computed radiation patterns at $2.4 \mathrm{GHz}$ for the two monopole microstrip patch fractal shaped antennas: (upper) E-plane pattern and (lower) H-plane pattern.

\begin{tabular}{|l|ll|l|l|}
\hline \multirow{2}{*}{ HFSS } & $\bullet$ & Sierpinski pentagon & \multirow{2}{*}{ Measured } & Sierpinski pentagon \\
\cline { 2 - 3 } & $\bullet \bullet .-$ Complementary Sierpinski pentagon & & Complementary Sierpinski pentagon \\
\hline
\end{tabular}
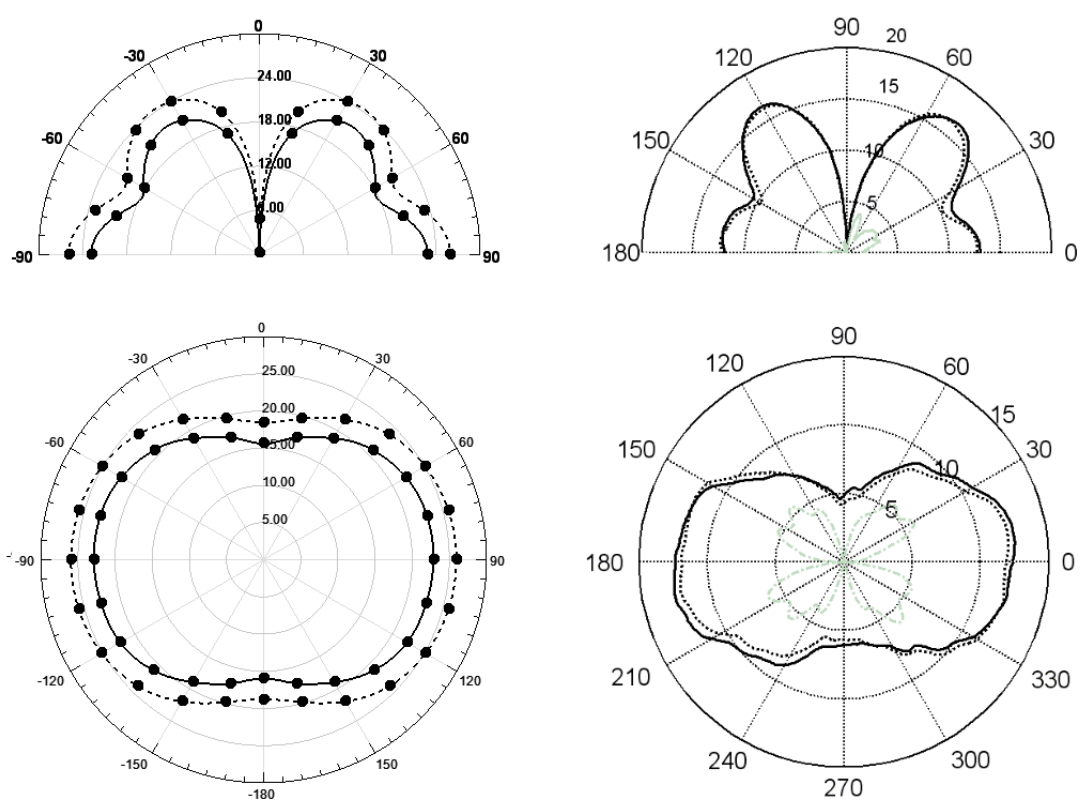

Fig. (7) Measured [7] and computed radiation patterns at $5.2 \mathrm{GHz}$ for the two monopole microstrip patch fractal shaped antennas: (upper) E-plane pattern and (lower) H-plane pattern. 
The 3D total gain patterns for the two fractal shaped antenna models at each band frequency were computed by the HFSS software and shown in Figs. (8) and (9). The difference in values of the radiation patterns that are present in the $2 \mathrm{D}$ plot are not noticeable in the $3 \mathrm{D}$ plot.
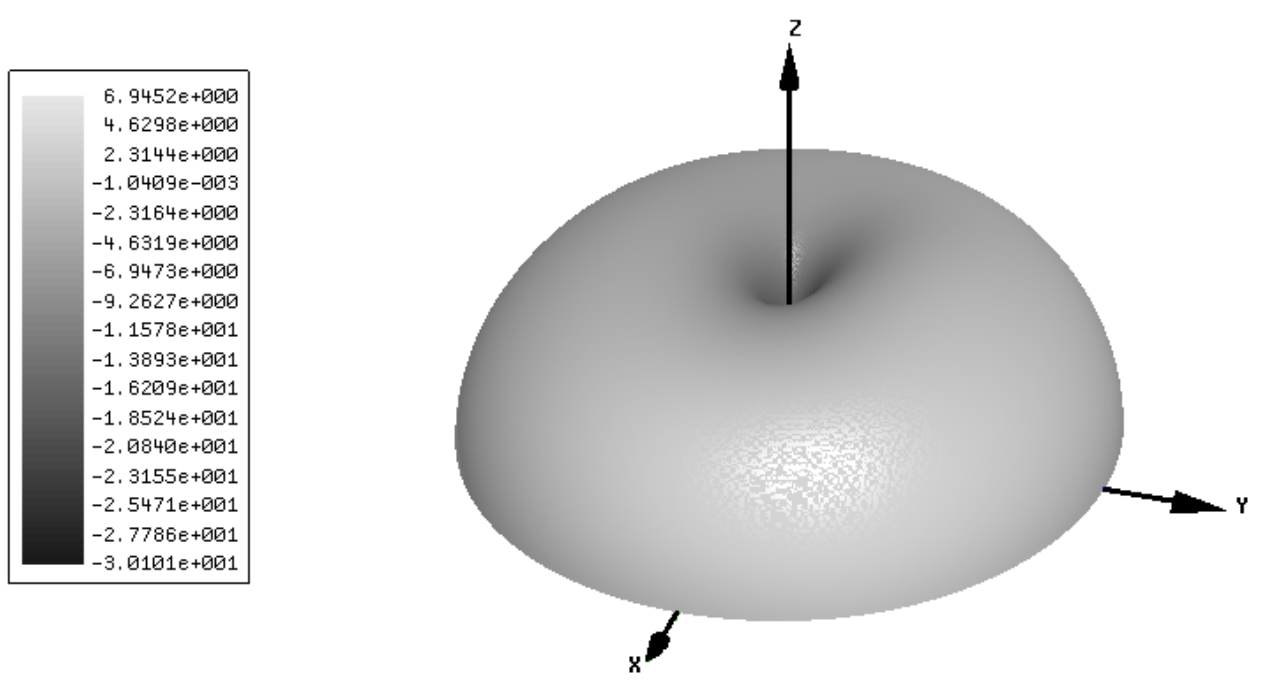

(a) Sierpinski pentagon
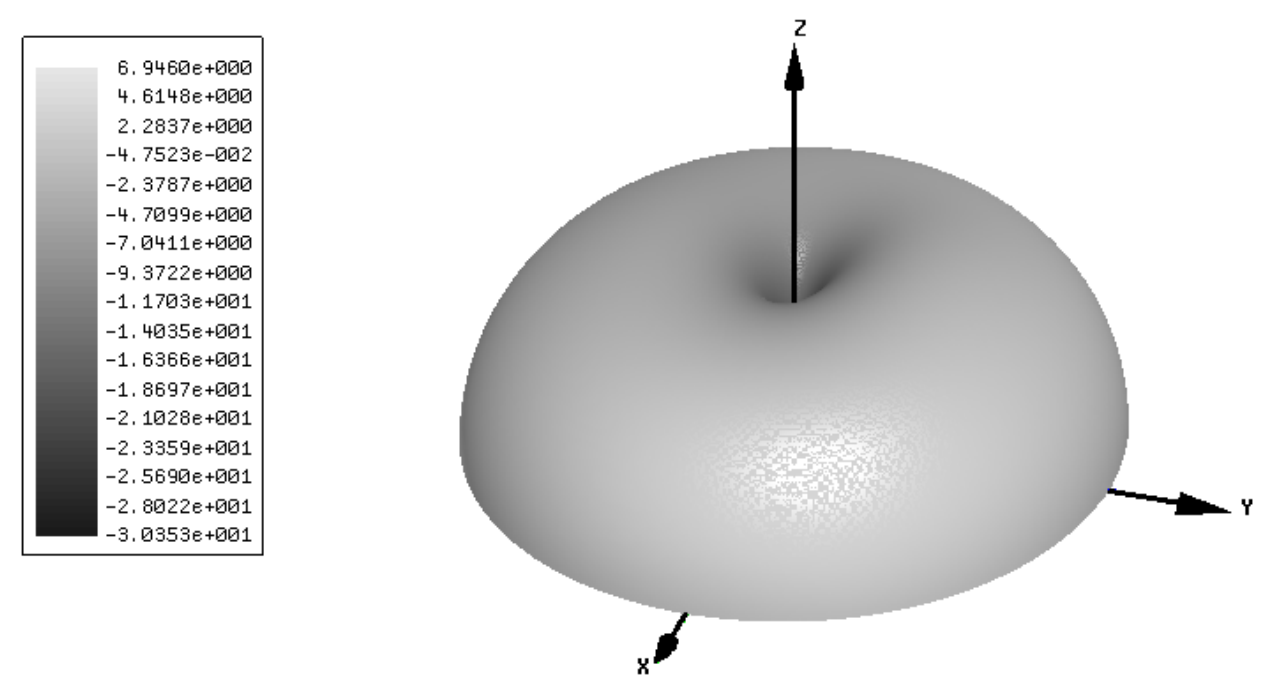

(b) Complementary Sierpinski pentagon

Fig. (8) 3D side view of the total gain in (dB) computed at $2.4 \mathrm{GHz}$ by the HFSS software for the monopole Sierpinski pentagon and monopole complementary Sierpinski pentagon microstrip patch fractal shaped antennas. 

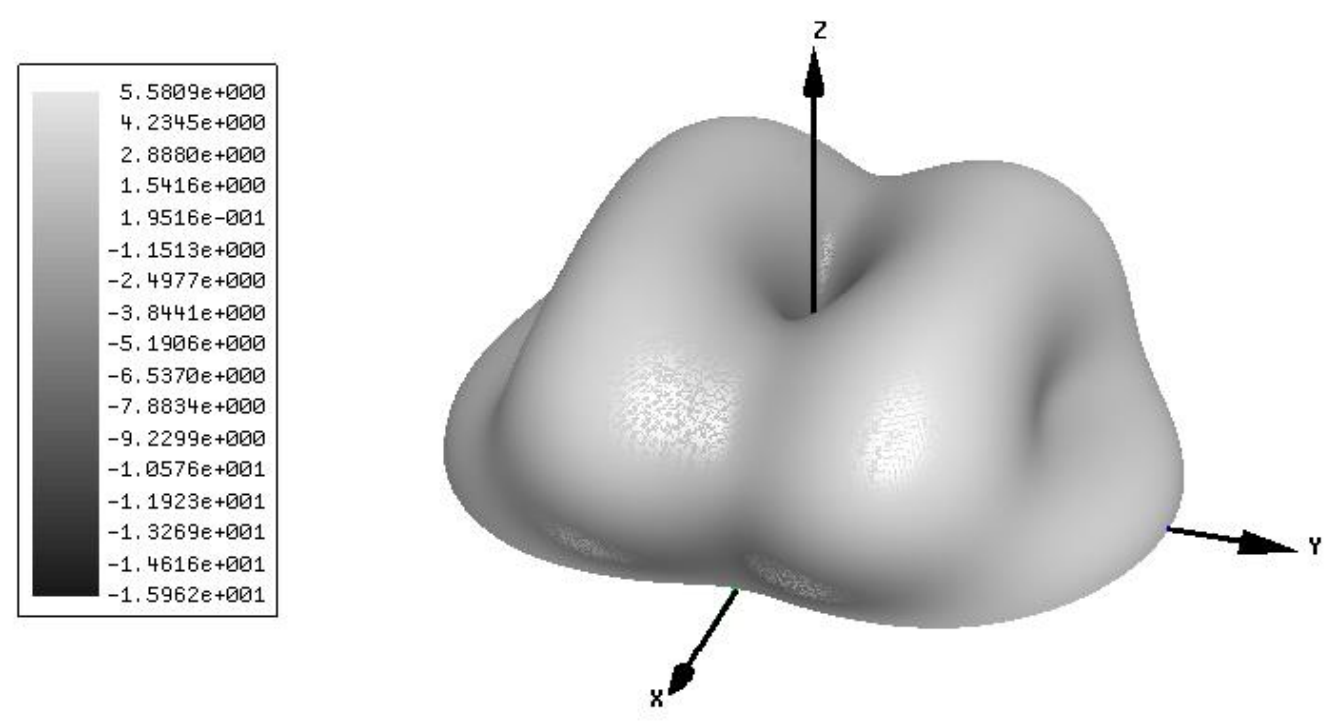

(a) Sierpinski pentagon
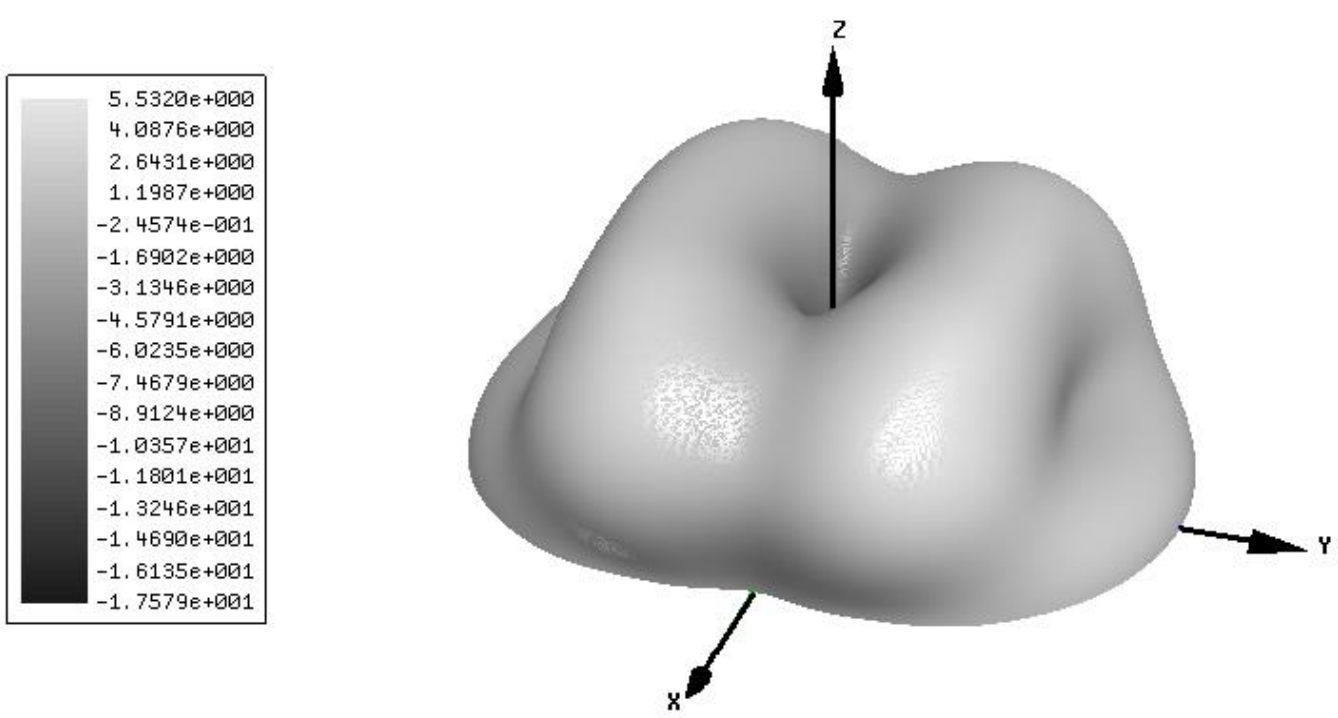

(b) Complementary Sierpinski pentagon

Fig. (9) 3D view of the total gain in (dB) computed at $5.2 \mathrm{GHz}$ by the HFSS software for the monopole Sierpinski pentagon and monopole complementary Sierpinski pentagon patch fractal shaped antennas. 
The $2.4 \mathrm{GHz}$ and $5.2 \mathrm{GHz}$ current distributions for the patches of both antennas are illustrated in Figs. (10) and (11) respectively. At $2.4 \mathrm{GHz}$, the locations of the hottest areas in both patches are similar to each other and exist near the feed ports. These hottest areas are excited to create the field lines with ground plane to complete their cycles. Therefore, the behavior of 3D total gain for both patches is the same and takes the shapes shown in Fig.(8). This also means that the 3D total gain patterns have symmetry around Z-axis. While at $5.2 \mathrm{GHz}$, there are three hottest areas located in both patches as shown in Fig.(11). The locations of these hottest areas are similar to each other for both patches. One of these areas is located near the feed port. The others are located near the boundaries of the patches from the feed side. All hottest areas are excited to create the field lines with ground plane to complete their cycle. The behavior of the 3D total gain for both patches is symmetric with respect to the XZ-plane as shown in Fig.(9). In this plane, the two lobes around the side of the $\mathrm{Z}$-axis are caused by the hottest area which is near the feed port; while the two lobes on $\pm \mathrm{Y}$-axis are caused by the two hottest areas which are located on the two sides of the patches.
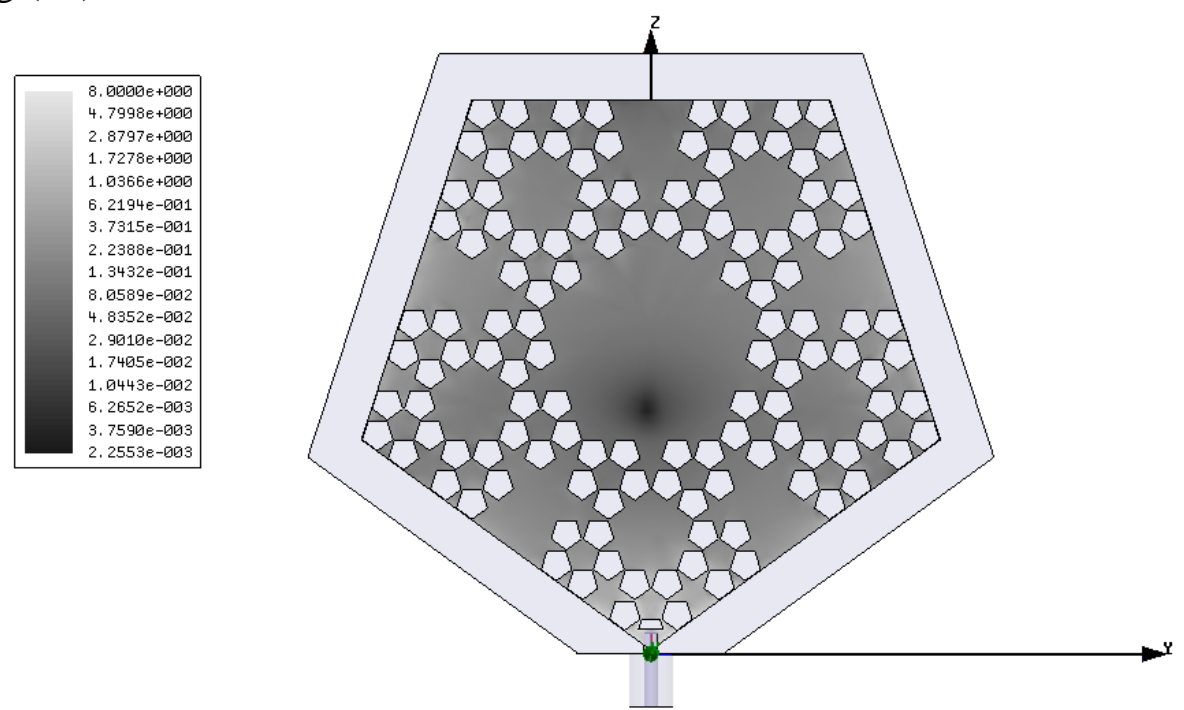

(a) Sierpinski pentagon
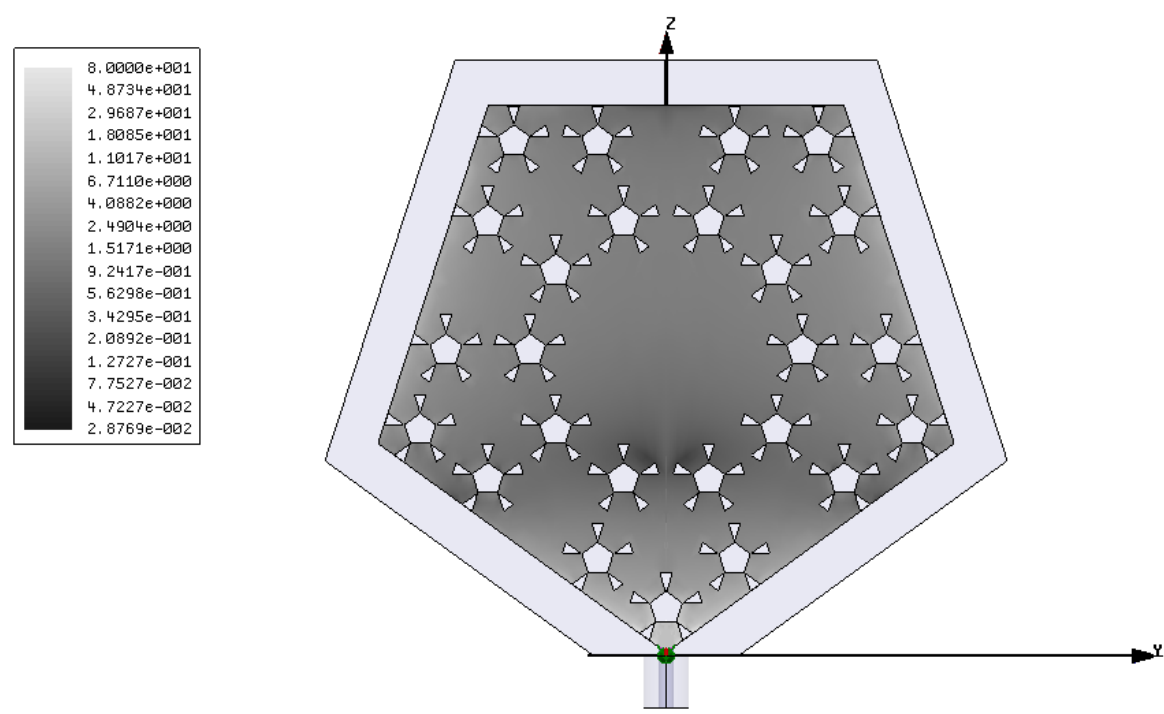

(b) Complementary Sierpinski pentagon

Fig. (10) Surface current (J) distribution with gray scale at $2.4 \mathrm{GHz}$ as computed by the HFSS software for the monopole Sierpinski pentagon and its complement microstrip patch fractal shaped antennas. 

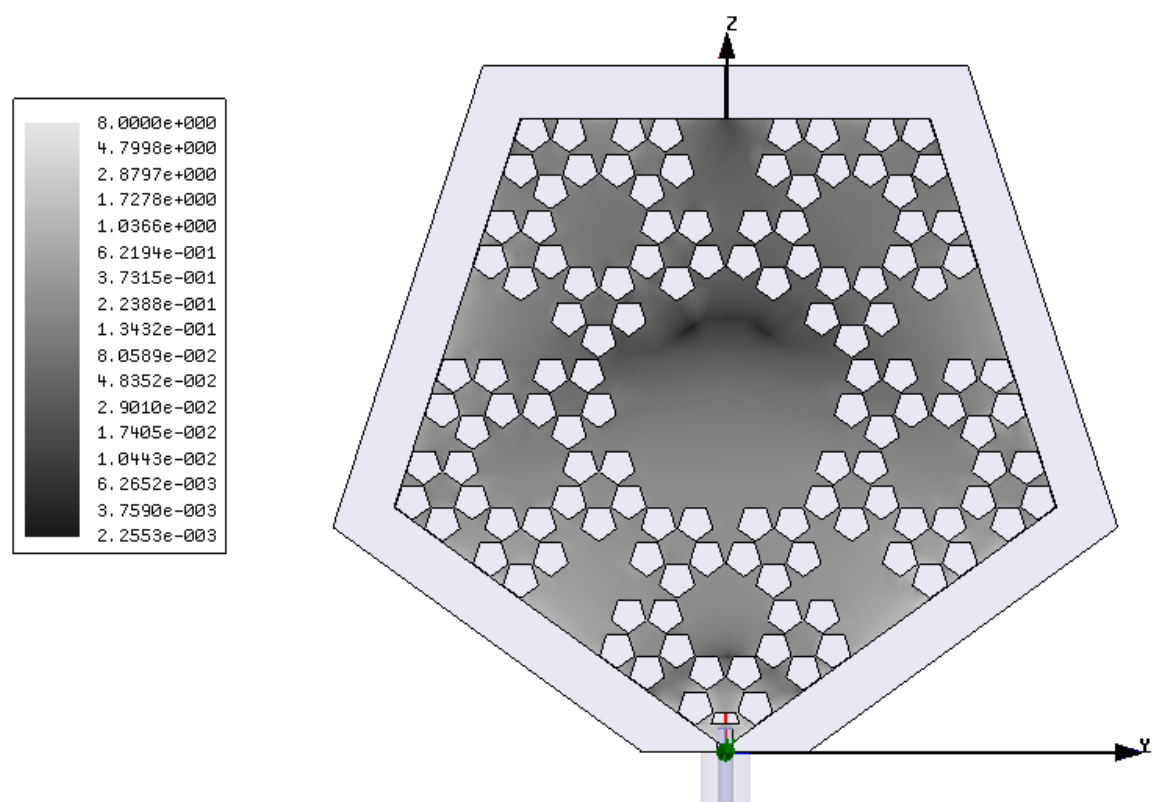

(a) Sierpinski pentagon
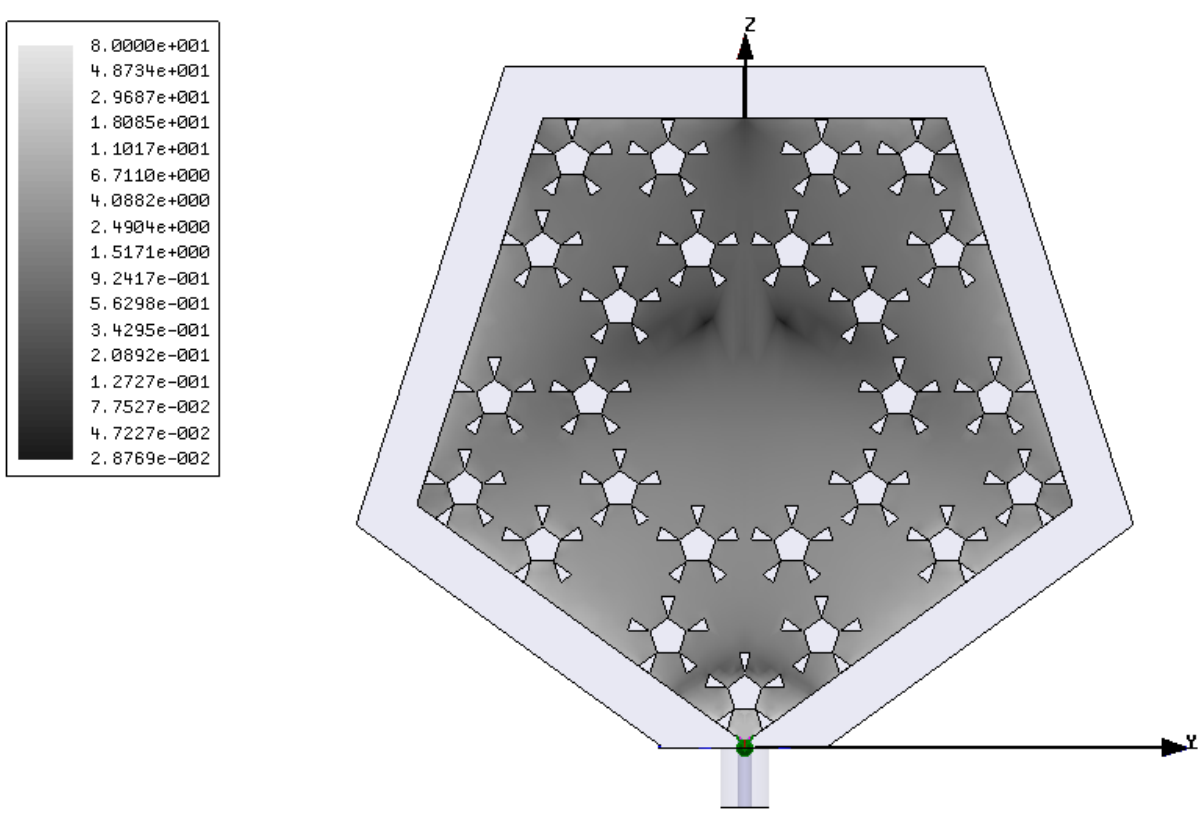

(b) Complementary Sierpinski pentagon

Fig. (11) Surface current (J) distribution with gray scale at $5.2 \mathrm{GHz}$ as computed by the HFSS software for the monopole Sierpinski pentagon and its complement microstrip patch fractal shaped antennas.

\section{Conclusions}

The present work confirms what has been found in previous published works that fractal shapes for antennas lead to improved performance in several aspects such as multiband behavior, miniaturization of physical size, better matching and enhanced directivity. Also, it shows that simulation of fractal shaped antennas in computer software helps in understanding the radiation pattern of these antennas, calculating many of their characteristics that cannot found by practical measurements, and comparing with measured data. This helps to understand the physical mechanisms that enter into play as a result of fractalization of antenna shapes.

The computed results obtained from the HFSS software for the two antennas show good agreement with measured data for the S-parameter and radiation patterns. Both 
antenna models show wideband behavior and good matching at the UMTS, Bluetooth, WLAN and HIPERLAN bands. Similar behavior for the variation of S-parameter in the studied frequency range is observed for both fractal shaped antennas in the computed results. This may be attributed to the fact that electric lengths are approximately the same for both antennas. In the Smith chart, there are four loops in the curve for the S-parameter for both fractal shaped antennas. Each loop represents a specific band in the rectangular plot. The loop shapes depend on the minimum value of the S-parameter and bandwidth. Taken altogether, the results of the present computations confirm that the two presently studied fractal shaped antennas have novel properties and warrant their use in real life applications.

\section{References}

[1] Mandelbrot, Benoit B. "The Fractal Geometry of Nature", W. H. Freeman, New York, 1993.

[2] Cohen, N. L. "Fractal Antennas: Part 1" Communications Quarterly, summer, 523, 1995.

[3] Romeu, J., Borja, C., Soler, J., Blanch, S., and Cardama, A. "Properties of Fractal Shaped Antennas" Antenna Theory and Measurement, Microwave Symposium, Tetuan, 398-401, May, 2000

[4] Hansen, Robert C. "Electrically Small, Superdirective, and Super-conducting Antennas", John Wiley and Sons Inc., Hoboken, New Jersey, 2006.

[5] Balanis, Constantine A. "Modern Antenna Handbook", John Wiley and Sons, Inc., New York, 2008.

[6] Volakis, John, Chen, Chi-Chih, and Fujimoto, Kyōhei, "Small Antennas: Miniaturization Techniques and Applications", McGraw-Hill, New York, 2010.

[7] Naghshvarian-Jahromi, M. and Komjani, N. "Novel Fractal Monopole Wideband Antenna", J. Electromagn. Waves Appl., 22, 195-205, 2008.

[8] 'User's Guide-High Frequency Structure Simulator", Software Version: 10.0, First Edition, Ansoft Corporation, Pittsburgh, USA, 2005.
[9] Shaalan, Amer Basim "Simulation of Fractal Antenna Properties Using Fractal Geometry", Ph.D. Thesis, Physics Department, Al-Mustansiriayah University, Baghdad, Iraq, 2002.

[10] Ataeiseresht, R., Ghohadi, Ch. and Nourinia, J. "A Novel Analysis of Minkowski Fractal Microstrip Patch Antenna", J. of Electromagn. Waves and Appl., 20, 1115-1127, 2006.

[11]Lui, Huilai, Wang, Bing-Zhong and Shao, Wei "Dual Band Bi-Directional Pattern Reconfigurable Fractal Patch Antenna for Milimeter Wave Application", Springer, International Journal of Infrared and Millimeter Waves, 28, 25-31, 2007.

[12] Yadava, R. L., Ram, M. and Das, S. "Multiband Triangular Fractal Antenna for Mobile Communications", International Journal of Engineering Science and Technology, 2, 6335-6348, 2010.

[13]Schlicker, Steven and Dennis, Kevin "Sierpinski n-gons" $\mathrm{Pi} \mathrm{Mu}$ Epsilon Journal, 10, 81-89, 1995.

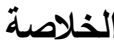

من المعروف أن القياسات المختبرية للهوائيات ذات الاشكال الكسورية غير كافية لفهم هذه الهوائيات وتصرفها الفيزيائي وهنالك خصائص لهذه الهوائيات لايمكن الحصول

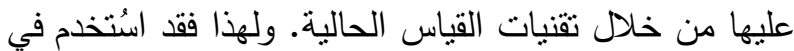
العمل الحالي البرنامج الحاسوبي Ansoft HFSS المعتمد على طريقة العناصر المحدودة (FEM) لحل المسائل الكهرومغناطيسية لنمذجة وتمثنيل وتحليل هوائي الرقعة المطبوعة احادي القطب ذي الثنل الكسوري (خماسي سربنسكي) ومكمله. اظهرت نتائج التمثيل الحاسوبي

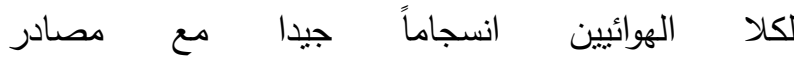
تغذيتهما في مدى الترددات (12 GHz - 2 GHz) وبينت انهما بصلحان للعمل في انطقة التردد لعMTS Bluetooth , $(2200 \mathrm{MHz}-2000 \mathrm{MHz})$ $(2.4 \mathrm{GHz})$ WLAN g (2480 MHz - $2400 \mathrm{MHz})$ و 5.2GHz) HIPERLAN). الحسابات بشكل عام اتفاقا جيدا مع نتائج القياسات لمعامل ونمط الاشعاع لهذين الهوائيين. 